https://helda.helsinki.fi

\title{
Luther und humanistische Philosophie
}

\section{Saarinen, Risto}

Vandenhoeck \& Ruprecht

2013

Saarinen , R 2013 , Luther und humanistische Philosophie . in Lutherjahrbuch . vol. 80 , Vandenhoeck \& Ruprecht, pp. 77-109 .

http://hdl.handle.net/10138/152697

acceptedVersion

Downloaded from Helda, University of Helsinki institutional repository.

This is an electronic reprint of the original article.

This reprint may differ from the original in pagination and typographic detail.

Please cite the original version. 


\title{
LUTHER UND HUMANISTISCHE PHILOSOPHIE
}

\author{
Risto Saarinen
}

1. Das Erbe von Junghans und seine Weiterführung

Vor einer Generation unternahm Helmar Junghans in seinem Buch Der junge Luther und die Humanisten eine neue Gesamtwertung des Humanismus in seiner Bedeutung für das Denken Martin Luthers. ${ }^{1}$ Während die ältere Forschung vor allem den Gegensatz zwischen Luther und Erasmus von Rotterdam betonte, richtet Junghans seine Aufmerksamkeit auf die vielen Beziehungen Luthers zu den humanistischen Strömungen in Erfurt und Wittenberg sowie auf die humanistischen Interessen seiner Studienfreunde.

Junghans hebt die große Bedeutung der Rhetorik und der humanistischen Auffassung von Sprache und Stil hervor. Die literarische und pädagogische Form der Schriften Luthers zeuge von einem positiven Einfluss des Humanismus auf den Reformator. Die Arbeitsweise Luthers sowie sein methodisches Vorgehen folgten oft der humanistischen Wissenschaftsmethode, die durch einen programmatischen Gegensatz zur herkömmlichen Scholastik gekennzeichnet sei. $^{2}$ Beim Aufzeigen dieser Ähnlichkeiten will Junghans sorgfältig vorgehen: Einerseits konstatiert er, dass auch Luthers inhaltliche Erkenntnisse einen Zusammenhang mit der humanistischen Denkrichtung aufweisen, andererseits will er die reformatorischen Einsichten nicht auf eine Geistesströmung reduzieren, sondern betont die Eigenart der theologischen Sprache und deren Inhalte. ${ }^{3}$

In den letzten 30 Jahren hat die Humanismusforschung beträchtliche Fortschritte genommen. Viele Forscher haben Spezialstudien veröffentlicht, in denen das Verhältnis zwischen Reformation und Humanismus detailliert untersucht wird. ${ }^{4}$ In meinem heutigen

${ }^{1}$ H. Junghans, Der junge Luther und die Humanisten, 1985.

${ }^{2} \mathrm{AaO} ., 269-273,319-323$.

${ }^{3} \mathrm{AaO} ., 323-325$.

${ }^{4}$ An dieser Stelle kann z.B. die Buchreihe „Spätmittelalter, Humanismus, Reformation“ (Tübingen: Mohr Siebeck) erwähnt werden, die seit 2008 die ältere Reihe „Spätmittelalter und Reformation“ ersetzt. Siehe auch E. RUMMELS zahlreiche 
Vortrag werde ich einige Züge der humanistischen Philosophie und sowie ihre Bedeutung für das Denken Luthers erläutern. Philosophiegeschichtliche Studien haben zu beweisen versucht, dass Renaissance und Humanismus als philosophisch eigenständige und kreative Perioden aufgefasst werden könnten. ${ }^{5}$ Mit deren Hilfe könnte behauptet werden, dass der Humanismus nicht nur wegen seines literarischen Stils und seiner antischolastischen Methode, sondern auch wegen seines philosophischen Gehalts für die Reformation bedeutsam war.

Zuerst sollen die neueren Anschauungen über die Eigenart von Renaissancephilosophie bzw. Philosophie im Humanismus präziser erläutert werden. Es ist heute unbestreitbar, dass die Erneuerung von Sprache und Methode ein zentrales Anliegen der Renaissancephilosophie war. Von den neuen Büchern kann Lodi Nautas Studie zur humanistischen Kritik der scholastischen Philosophie erwähnt werden. ${ }^{6}$ Durch eine sorgfältige Analyse von Lorenzo Vallas Repastinatio dialectice et philosophie weist Nauta nach, wie die Humanisten das technische Vokabular der Scholastik durch eine allgemein verständliche Sprache ersetzen. Auf diese Weise werden zum Beispiel viele aristotelische Kategorien einfach als Qualitäten interpretiert. Des Weiteren werden die Tugenden nicht anhand der Habituslehre, sondern im Rahmen der Emotionstheorie als Affekte behandelt. Auch in der Trinitätslehre lehnt Valla manche aristotelische Kategorien ab, so dass die Trinität eher als Mysterium denn als ein logisch-philosophisches Denkgebäude erscheint. ${ }^{7}$ Solchen im Grunde genommen sprachlichen und methodischen Erneuerungen begegnen wir auch bei den Reformatoren; so beweist Nautas neue Studie, dass die Beobachtungen von Junghans hinsichtlich der humanistischen Sprache und Methode treffend und aktuell geblieben sind. Die neue Studie von William Wright zeigt im Weiteren, dass der Einfluss von Lorenzo Valla auf Luther erneut brisant geworden ist. ${ }^{8}$

Veröffentlichungen sowie das neue Buch von W. WRIGHT, Martin Luther's Understanding of God's Two Kingdoms, 2010.

${ }^{5}$ Siehe z.B. B. COPENHAVER - C. SCHMitT, Renaissance Philosophy, 2003; J. HANKInS (ed.), The Cambridge Companion to Renaissance Philosophy, 2007 und R. SAARINEN, Weakness of Will in Renaissance and Reformation Thought, 2011.

${ }^{6}$ L. NAUTA, In Defense of Common Sense: Lorenzo Valla's Humanist Critique of Scholastic Philosophy, 2009.

\footnotetext{
${ }^{7}$ AaO., 82-128, 152-210.

${ }^{8}$ Wright (s. Anm. 4).
} 
Die große Frage der Renaissancephilosophie ist aber, ob die Erneuerung in Sprache und Methode auch neue inhaltliche Schwerpunkte und philosophische Fragestellungen mit sich bringt. Viele Studien beantworten diese Frage eher negativ und zeigen, wie der mittelalterliche Aristotelismus trotz des eleganten Lateins und der neuen Griechischkenntnisse relativ unverändert bleibt. Die von Quentin Skinner und Charles Schmitt herausgegebene umfassende Geschichte der Renaissancephilosophie zeigt die ungebrochene Kontinuität des Aristotelismus vom Mittelalter bis zum Zeitalter des Descartes. Die im Grunde genommen thomistischen philosophischen und theologischen Positionen werden in der Renaissance zwar mit elegantem Latein vertreten, aber neue inhaltliche Gesichtspunkte kommen eher selten zum Vorschein. ${ }^{9}$

Auf der anderen Seite werden in der neueren Forschung auch Positionen vertreten, die in der Renaissancephilosophie grundlegende inhaltliche Erneuerungen feststellen. Der französische Philosoph Pierre Hadot hat eine große Diskussion ausgelöst mit seiner Darstellung der Philosophie als Lebenskunst oder Lebensform. ${ }^{10}$ In seinen Studien vertritt Hadot die These, dass seit der Antike neben der theoretischen akademischen Philosophie eine andere Art von Philosophie existiert, die die Weisheit als praktischen Lebensweg oder eine Lebenskunst betrachtet. Diese andere Philosophie kann die Form von Therapie oder geistiger Übung annehmen; oft hat sie die Kontrolle oder Mäßigung der Emotionen zum Zweck. Hadot folgend haben viele Philosophiehistoriker mit unterschiedlichem Erfolg nach Spuren der therapeutischen Philosophie in verschiedenen Perioden der Ideengeschichte gesucht. ${ }^{11}$ Die Renaissance und der Humanismus eignen sich besonders gut für die Suche nach Philosophie als Lebenskunst. Weit verbreitete und viel gelesene Werke wie Petrarcas De remediis utriusque fortunae, die blühende Ars moriendi-Literatur ${ }^{12}$ oder viele Schriften von Erasmus von Rotterdam können im Rahmen des Programms „Philosophie als Lebenskunst“

${ }^{9}$ C. SCHMITT \& Q. SKInNER (ed.), The Cambridge History of Renaissance Philosophy, 1988. Ähnlich auch D. LINES, Aristotle's Ethics in the Italian Renaissance (ca. $1300-1650), 2002$.

${ }^{10}$ Besonders die englische Ausgabe: Philosophy as a Way of Life, 1995 (französische Texte teilweise schon früher).

${ }^{11} \mathrm{Zu}$ den ersten Versuchen gehört M. Nussbaum, The Therapy of Desire: Theory and Practice in Hellenistic Ethics, 1994. Siehe auch R. SORABJI, Emotion and Peace of Mind, 2000.

${ }^{12}$ Zu Petrarca z.B. SAARINEN, Weakness (s. Anm. 5), 43-54; zu Ars moriendi B. HAMM, „Luthers Anleitung zum seligen Sterben vor dem Hintergrund der 
interpretiert werden, da sie von der Wirkungskraft der philosophischen Gedanken außerhalb des akademischen Vorlesungsraums zeugen. Im Prinzip könnte man die Eigenart der Philosophie in der Renaissance bzw. im Humanismus als neue Popularität der Philosophie als Lebensform definieren. ${ }^{13}$

Näher betrachtet eignet sich Hadots Ansatz allerdings kaum als alternative Gesamterklärung der Philosophiegeschichte. Sein Ansatz kann zwar zeigen, wie die Populärphilosophie am Rande der akademischen Philosophie existiert, aber die therapeutischen Alternativerklärungen folgen letzten Endes weitgehend den Ansätzen der akademischen Philosophie. ${ }^{14}$ Obwohl der Ansatz von „Philosophie als Lebensform“ deswegen keine grundlegend neuen Perspektive bietet, kann er als heuristische Einsicht in gewissen Themen hilfreich sein. Im Folgenden werde ich eine solche Einsicht auf dem Bereich der Lutherforschung verwenden, um gewisse Themen von humanistischer Philosophie sichtbar zu machen.

Luthers antischolastischer Ansatz trägt bisweilen populärphilosophische Züge, die durch den Humanismus geprägt sind. Die historischen Quellen solcher Populärphilosophie sind vor allem Ciceros De officiis und Senecas De beneficiis, die zu Luthers Zeiten weit über die Universitäten hinaus verbreitet waren und als Lehrbücher des angemessenen Verhaltens gelesen wurden. ${ }^{15}$ Wahrscheinlich erscheint die Populärphilosophie von Cicero und Seneca für Luther in erster Linie als allgemeines Kulturerbe und soziales Verhaltensmuster, das in den gebildeten Kreisen in Luthers Umgebung ${ }^{16}$ bekannt war. Wenn Luther Motive aus dieser Tradition gebraucht, geht es für ihn vor allem um die Kommunikation theologischer Sachverhalte: Wenn er seine Theologie anhand der populären Verhaltensmuster beleuchtet,

spätmittelalterlichen Ars moriendi““ (in: ders., Der frühe Luther, 2010, 115-163).

${ }^{13}$ So z.B. S. EBBERSMEYER, „Philosophie als Lebensform: eine Einführung in die Philosophie der Renaissance“, www.phil-hum-ren.uni-muenchen.de/LV/EinfWise99.htm (gelesen 19.12.2012).

${ }^{14}$ Mein eigenes Urteil folgt an dieser Stelle den materialreichen Studien von SORABJI, Emotion (s. Anm. 11) sowie ders., Self: Ancient and Modern Insights about Individuality, Life, and Death, 2006.

${ }^{15}$ Siehe z.B. J. KRAYE, „Moral Philosophy“ (in: SCHMitT \& SKInNER, Cambridge History (s. Anm. 5), 301-386). Zu Senecas theologische Bedeutung vgl. T. ENGBERGPEDERSEN, „Gift-Giving and Friendship: Seneca and Paul in Romans 1-8 on the Logic of God's Charis and Human Response“ (HTR 101, 2008, 15-44) sowie M. HENAFF, Der Preis der Wahrheit, 2009, 393-409 (franz. Original 2002).

${ }^{16}$ Hilfreich für die Identifizierung dieser Kreise ist L. GRANE, Martinus noster: Luther 
verstehen seine Leser und Zuhörer seine Botschaft besser mit Hilfe dieses Sprachgebrauchs. Luthers Botschaft ist aber an sich eine theologische.

Im Folgenden werde ich die über Junghans hinaus führende These vertreten, dass Luther vom Humanismus nicht nur Sprache und Methode, sondern darüber hinaus gewisse populärphilosophische Inhalte übernimmt, die bei Cicero und Seneca vorhanden sind und Luthers Publikum allgemein bekannt waren. Wenn ich diesen Schritt wage, behaupte ich nicht, es hätte zu Luthers Zeiten so etwas wie eine „Philosophie als Lebenskunst“ im Sinne von Pierre Hadot gegeben. Ich kann im Folgenden keinen systematischen Vergleich zwischen Luther einerseits und Cicero und Seneca andererseits anstellen; ich gehe davon aus, dass es sich bei den festgestellten Ähnlichkeiten eher um ein gemeinsames Kulturerbe des Humanismus handelt als um eine unmittelbare literarische Abhängigkeit. ${ }^{17}$

$\mathrm{Zu}$ dieser Auffassung haben mich zwei neue Studien geleitet, die dieses gemeinsame Kulturerbe sowohl sozialgeschichtlich als auch philosophiegeschichtlich beleuchten. In ihrem Buch The Gift in Sixteenth-Century France untersucht Natalie Zemon Davis die mannigfaltigen Gabenbeziehungen im frühneuzeitlichen Europa. Nach ihr konnten die Menschen im 16. Jahrhunderts sehr scharfsinnig und nuanciert zwischen Gabenbeziehungen einerseits und Handelsbeziehungen andererseits unterscheiden. Das angemessene Geben und Empfangen von Gaben war in allen sozialen Klassen ausgesprochen wichtig, da Ehre, Rang und soziale Wertschätzung nicht in erster Linie gekauft und verkauft, sondern mit gegenseitigen Gaben manifestiert werden konnten. ${ }^{18}$ Für die oberen Klassen wurden die bestehenden Rangordnungen deswegen anhand von Gaben konkretisiert; Seneca und Cicero hatten die Natur solcher Gaben philosophisch bestimmt. Auch für die armen Menschen waren

in the German Reform Movement 1518-1521, 1994.

${ }^{17}$ Trotz einiger positiver Äußerungen LUTHERS über De officiis (e.g WATR 2, 456, 29 - 457, 3) bietet das WA-Sachregister kaum ein informatives Bild von Luthers Gebrauch von Cicero und Seneca. Man sollte vor allem die Begrifflichkeit wie honestum, beneficium, gratitudo, gratias agere, consuetudo, dignus usw. sowie die bei Cicero und Seneca vorhandenen Argumente über das rechte Geben und Empfangen von Wohltaten, Gaben usw. sorgfältig mit Luther vergleichen. Z.B. diskutieren CICERO (off 1, 49) und SENECA (ben 1, 5$7,2,18-25,31-35)$ ausführlich die Natur von ,accepta beneficia“. Diese Wendung kommt auch bei LUTHER häufig und an theologisch prägnanten Stellen vor, z.B. WA 3, 89, 12; 276, $17 ; 5,181,18 ; 6,364,20 ; 13,355,23 ; 20,33,29 ; 29,452,2 ; 454,9-13$. Die Rede von einem gemeinsamen „Kulturerbe“ soll also die literarische Abhängigkeit nicht allzu schnell ausschließen.

${ }^{18}$ ZEMON DAVIS, The Gift in Sixteenth-Century France, 2000, 56-73 (auf S. 59-61 
Gaben belangvoll, weil sie ihre sozialen Beziehungen nur bisweilen mit Geld und Handel regulieren konnten; oft waren es eher gegenseitige Wohltaten und Geschenke, aus denen das soziale Leben in der Gesellschaft bestand.

Auch für das Gottesverhältnis und die Religion war das rechte Unterscheiden zwischen Gabenbeziehungen und Handelsbeziehungen ausschlaggebend. Die Reformation hat zielgerichtet gegen die Irrlehre gepredigt, dass Gottes Gunst anhand von menschlichen Verdiensten gekauft werden könnte. Zemon Davis konstatiert wie folgt:

the religious reformations of the sixteenth century were a quarrel about the gifts, that is, about whether humans can reciprocate to God, about whether humans can put God under obligation, and about what this means for what people should give to each other. The religious quarrel stands on its own and is argued in its own terms, but is parallel to the quarrel about the king's obligation to his subjects. ${ }^{19}$

In seiner Studie Der Preis der Wahrheit erläutert Marcel Henaff die Ideengeschichte des Gedankens, dass die Wahrheit und die rechte Lehre keinen Preis haben, da sie nicht zum Privatbesitz des Individuums gehören und deswegen nicht gekauft oder verkauft werden können. Wahrheit, Lehre und Unterweisung gehören darum eher in den Bereich der Gabenbeziehungen als in den der Handelsbeziehungen. Die Gegenseitigkeit, die für das historische Weitergeben von Lehre und Wahrheit notwendig ist, ist darum eine Gegenseitigkeit der Anerkennung, Ehre und Dankbarkeit, die durch die angemessene Gabenbeziehung entsteht. ${ }^{20}$ Auch für Henaff erscheint die Reformation als Kampf um die rechte religiöse Bestimmung von Gabe, Dankbarkeit, Leistung und Preis. Er schreibt: „Wahrscheinlich ist es letztlich die Frage der Gabe, die die beiden im christlichen Abendland herrschenden Konfessionen trennt.“21

Henaff geht es grundsätzlich darum, dass ,„der vertraglich geregelte Handelsaustausch“ und die dazu gehörende Rationalität erst mit der Reformation zur herrschenden Norm des alltäglichen, weltlichen Lebens werden. Im mittelalterlichen Christentum manifestierte die Mildtätigkeit der guten Werke teils die Gabenbeziehungen, teils die Handelsbeziehungen, die

diskutiert sie das Verhalten von Erasmus).

${ }^{19} \mathrm{AaO} ., 167-168$.

${ }^{20}$ HeNAFF, Preis (s. Anm. 15), 36-40. Wie er auf S. 539-552 erläutert, hatte die Entdeckung des Buchdrucks und der entstehende Beruf des Buchhändlers diese Einsicht von Lehre und Wahrheit verändert.

${ }^{21} \mathrm{AaO}, 411$. 
stets als miteinander gemischt erschienen. Der theozentrische Protestantismus hat eine klare Trennung zwischen den beiden etabliert, indem er die Gabe auf Gottes Wirksamkeit zurückführte und die Möglichkeit der menschlichen guten Werke bestritt. So wurden im Protestantismus die Gabenbeziehungen rein innerlich und theologisch verstanden, während die entzauberte, äußerliche Welt anhand der Handelsbeziehungen reguliert wurde. ${ }^{22}$

Weder Henaff noch Zemon Davis interpretieren Luthers eigene Texte, aber sie behandeln ausführlich Luthers geistige Umgebung. Im Folgenden werde ich einige Texte Luthers zu theologischen Gabenbeziehungen interpretieren. Drei Themenbereiche kommen vor, erstens die Natur der Wohltaten, zweitens die Art der rechten Lehre und Unterweisung und drittens die Kunst des rechten Empfangens. Die angemessene Ehre und Dankbarkeit sowie das Vermeiden der Hochmut und die angemessene Demut werden stets im Kontext dieser Themen diskutiert. Parallelen aus Ciceros De officiis und Senecas De beneficiis werden erwähnt. Wir werden einerseits sehen, dass für Martin Luther die Unterscheidung von Gabenbeziehungen und Handelsbeziehungen durchaus belangvoll ist. Insofern bestätigt meine Diskussion die Thesen von Zemon Davis und Henaff. Andererseits zeigt Luther eine tiefere Sensibilität für diese Unterscheidung, als was Henaffs schematische Zweiteilung zwischen Protestantismus und Katholizismus annimmt.

\section{Beneficia - die Wohltaten Gottes}

Zuerst sollen drei Begriffe kurz erläutert werden: Gabe, Geschenk und Wohltat. Für Luther gehören die Gaben mit dem Glauben und der Gnade zusammen, während Verdienste und Werke eine Handelsbeziehung ausdrücken. So ist für Luther das Maß des Glaubens ein anderes als das Maß der Verdienste. Das antike Prinzip ,jedem das Seine“ kann aber auch als Prinzip der Gabenbeziehungen erscheinen, und so nehmen die Gabenbeziehungen an einer ihrem Maß entsprechenden Reziprozität teil. ${ }^{23}$

${ }^{22}$ AaO., 428-429.

23 Z.B. WA 17/1, 38, 12-23: „Das der glaube mit sich bringe als ein heubt gutt die andern gaben, das es darumb heysse glaubens mas und nicht unsers willens odder verdiensts mas, das wyr solche gaben nicht verdienet haben, sondern wo glaube ist, da ehret Gott den selben glauben mit ettlichen gaben als zur mitgabe odder ubergabe wie viel er will, als er spricht 1. Cor. 12.: 'Er teylet eynem iglichen aus nach seynem willen'. Eph. 4.: 'eyn iglich 
Den Grundbegriffen der Handelsbeziehung, also kaufen und verkaufen, entsprechen in der reziproken Gabenbeziehung das Geben und Empfangen von Wohltaten. Luther übersetzt den lateinischen Begriff beneficium stets mit „Wohltat“ (in Luthers Rechtschreibung: Wolthat). Als Substantivform des Gebens umfasst der Begriff „Gabe“ (donum) alle Primärobjekte des Gebensaktes. Die Gabe unterscheidet sich vom Handelsobjekt dadurch, dass sie keinen Preis hat und darum in der Reziprozität, die ihrem Maß entspricht, nur Dankbarkeit oder gar nichts als Gegenleistung verlangt. Im Akt des Gebens wird die Gabe allerdings nicht notwendigerweise zum Privatbesitz des Empfängers; zum Beispiel als Leihgabe kann die Gabe im Besitz des Gebers bleiben.

In Luthers Sprache implizieren Wendungen wie „Gnade geben“ somit keinen notwendigen Transfer in den Eigentumsverhältnissen: Auch im Menschen kann die Gnade immer noch Gottes Gnade bleiben. Analog dazu können Luthers Wendungen wie „Gottes Gabe“, „hohe“ bzw. „,köstliche Gabe“،24 so verstanden werden, dass die Gabe ein Eigentum des Gebers oder zumindest unbestimmt oder unverfügbar bleibt - semantische Möglichkeiten, die für die nähere Bestimmung von einigen theologischen Gaben höchst bedeutsam sind. Wenn der Eigentumstransfer oder zumindest ein Verwaltungstransfer im Akt des Gebens unterstrichen wird, kommt das Wort „ubergeben“ in Luthers Sprache oft vor. ${ }^{25}$

Der Begriff Geschenk, der bei Luther ebenfalls oft vorkommt, häufig in der doppelten Wendung „Gabe und Geschenk“, ist bisweilen mit Gabe synonym. Im Begriff Geschenk ist allerdings das Eigentumsverhältnis stärker mit dem Empfänger verbunden. ${ }^{26}$ Wenn Luther zum Beispiel sagt: „,eyn gabe und geschenck, das dyr von Gott geben, und deyn eygen sey“227, verbindet er durch den zitierten Relativsatz das Moment des Gebens mit „Gabe“ und das

gelied ynn seyner mas'. Eben darumb spricht er auch, es seyen mancherley gaben, nicht nach unserm verdienst, sondern nach der gnaden, die uns geben ist, Das also die gnade gleich wie der glaube mit sich bringe solch edle kleynot und geschencke, eym iglichen seyne mas, Das ia also allenthalben die werck und verdienst ausgeschlossen und wyr mit den wercken nuer zum nehisten geweyset werden.“

${ }^{24}$ Z.B. Gottes Gabe: WA 16, 299, 34-37; hohe Gabe: WA 51, 215, 24; köstliche Gabe: WA 20, 392, 3 (Anm.). In WA 50, 368, 17-18 wird Musik als „,koestliche Gabe Gottes" bezeichnet; diese Gabe bleibt offenbar nicht im Privatbesitz des Empfängers.

${ }^{25}$ Wie z.B. WA 52, 620, 1-16 im Anschluss an Matth. 11, 27 zeigt, ist ,ubergeben“ die Wiedergabe vom griechischen paradidômi, ein Wort, das viele theologische Bedeutungen trägt. Siehe dazu R. SAARINEN, God and the Gift, 2005, 37-44.

${ }^{26}$ Auch im heutigen Deutsch wird darum z.B. „Leihgeschenk“ viel seltener gebraucht als „Leihgabe“ 
Moment des Besitzes mit „Geschenk“. Ähnliches geschieht in Wendungen wie: „gabe und geschenck ...von Gott mitgeteilt und verliehen“ oder „gab und geschenck ... Das du magst sagen: kom her, lieber herr, Du bist mein““ ${ }^{28}$ In diesen Wendungen unterscheidet Luther zwischen der Dynamik des Gebens (von Gott geben, mitgeteilt, kom her: Gabe) einerseits und dem resultierenden Eigentumsverhältnis (deyn eigen, verliehen, bist mein: Geschenk) andererseits.

Wenn Luther das Wort „Geschenk“ benutzt, fügt er auch häufig eine Eigentumsbezeichnung wie ,zu eigen“ oder „,mein eigen“ hinzu. ${ }^{29}$ So hat im „Geschenk“ der Transfer zum Privatbesitz des Empfängers deutlicher stattgefunden als in der allgemeinen „Gabe“. Alle Geschenke sind folglich auch Gaben, aber nicht alle Gaben sind Geschenke; zum Beispiel kann der Sonnenschein als Gabe bezeichnet werden, aber er kann kein solches Eigentum werden, das ihn zu einem Geschenk macht. Folglich ist die Wendung ,ich erhielt es als Gabe und Geschenk“ nicht tautologisch, sondern sie unterstreicht, dass der Gegenstand sowohl (a) mir gegeben als auch (b) zu meinem konkreten Eigentum geworden ist. Auf diese Weise entspricht „Geschenk“ dem lateinischen Begriff donatio. ${ }^{30}$

Der Begriff der Wohltaten, beneficia, hat einen wichtigen Stellenwert für Humanisten wie Erasmus von Rotterdam und Philipp Melanchthon. Darum haben die Forscher völlig richtig den Hintergrund des Begriffs im Humanismus lokalisiert. ${ }^{31}$ Auch Luther gebraucht diesen Begriff sehr häufig, aber die Lutherforscher haben merkwürdigerweise die Beziehungen zwischen Luther und humanistischer Philosophie nicht anhand dieses Begriffs untersucht. In

${ }^{27}$ WA $10 / 1,1,11,13-14$.

${ }^{28}$ WA 16, 353, 31; WA 49, 661, 41- 662,23.

${ }^{29} \mathrm{Zu}$ diesem Phänomen siehe M. SEILS, „Gabe und Geschenk: eine Zugabe“ (in: Denkraum Katechismus, hg. von J. von LÜPKE und E. THAIDIGSMANN, 2009, 87-103), hier: 98-99.

${ }^{30}$ Diese Beobachtungen möchte ich Martin Seils` äußerst wertvollen Studien zu Gabe und Geschenk in der Luthersprache hinzufügen. Es ist merkwürdig, dass Seils diese Unterscheidung zwischen Gabe und Geschenk schließlich nicht macht, obwohl schon seine allererste Belegstelle (WA 10/1, 1, 11, 12-18, S. 87) sie expliziert. SEILS (aaO., 87) spürt auch korrekt, dass bei Luther hinter „Geschenk“ der rechtliche Begriff „donatio“ steckt, vgl. z.B. WA 4, 596, 13-14: „Quia donatio, dicunt Iuristae, constituit possessorem recipientem ..." - Schon in seinem Hauptvortrag auf dem Lutherforschungskongress in Erfurt 1983 hat Seils Luthers Theologie als Theologie des Gebens bezeichnet, s. M. SEILS, „Die Sache Luthers" (LuJ 52, 1985, 64-80).

${ }^{31}$ Siehe z.B. H. G. PÖHLMAnNs Anmerkungen in P. MelanCHTHON, Loci communes 1521, Lateinisch-Deutsch, 1993, 21-22. 
einer längeren Studie habe ich neulich nachgewiesen, dass alle wichtigen Dimensionen von beneficia bei Luther schon in seiner ersten Psalmenvorlesung vorhanden sind. ${ }^{32}$ Diese Beobachtung zeigt erstens, dass Luther den Begriff nicht erst von Melanchthon gelernt hat. Zweitens unterstützt sie die These von Helmar Junghans, der zufolge schon der junge Mönch Luther wichtige Anstöße vom Humanismus bekommen hat.

Im Folgenden werde ich nur einige Abschnitte aus der ersten Psalmenvorlesung hervorheben und sie mit dem wichtigsten humanistischen Quellentext, Senecas De beneficiis, kurz vergleichen. Der grundlegende Unterschied zwischen Gabenbeziehungen und Handelsbeziehungen prägt das Verständnis von Wohltaten bei Luther und Seneca Für beide ist darüber hinaus der Unterschied zwischen göttlichen und menschlichen Wohltaten charakteristisch.

Ein gerechter Akt, konstatiert Luther, ist durch die angemessene Berücksichtigung von Verdiensten und Schulden charakterisiert. Dagegen berücksichtigt eine Wohltat die Verdienste des Empfängers nicht. ${ }^{33}$ Unter den Menschen werden Wohltaten durch andere Wohltaten belohnt; auch die Heiden können Wohltaten auf diese Weise in den Kreislauf bringen. Gott kann allerdings Wohltaten auf eine radikalere Weise hervorbringen, nämlich so, dass er allen Menschen Wohltaten erweist, ohne Rücksicht auf Dankbarkeit. Gottes Sein ist im Geben, so dass er Gutes austeilt und somit sogar Böses mit Gutem vergilt. Gott erscheint als wahrer Gott, indem er ausschließlich gibt und die Menschen ausschließlich empfangen. Um dieses wahre Gott-Sein aufrechtzuerhalten, nimmt Gott keine Wohltaten von den Menschen entgegen. ${ }^{34}$ Auf diese Weise bleibt Gott außerhalb des Kreislaufs der Wohltaten; zugleich ist er aber das Vorbild und der Ansatzpunkt aller menschlichen Wohltaten.

Ein Mensch soll die Wohltaten Gottes empfangen und Dank sagen. Im Akt des Empfangens erkennt der Mensch an, dass alles Gute im Leben von Gott gegeben ist. ${ }^{35} \mathrm{Im}$ Einklang mit diesem Gedanken konstatiert Luther, dass Gott schon im natürlichen Lauf des Lebens den Menschen täglich verschiedene Wohltaten erweist, wie Leben, Sein, Fühlen, Geist, Essen, Kleidung, Sonne, Himmel und Erde sowie alle Elemente. Darüber hinaus gibt

${ }^{32}$ R. SAARINEN, „Luther und Beneficia“ (in: The Reformation as Christianization, ed. A. M. JOHNSON \& J. A. MAXFIELD (SHR 66), 2012, 169-188).

${ }^{33}$ WA 55/2, 888, 6-13.

${ }^{34}$ WA 55/2, 883, 100-103; 889, 18-36.

${ }^{35}$ WA 55/2, 105, 10-14; 889, 18-21. WA 55/1, 660-661. 
Gott verschiedene geistliche Wohltaten, wie Essen und Kleidung in geistlichen Sachen, Sonne der Gerechtigkeit und alle Wohltaten der Kirche. ${ }^{36}$

Diese Grundzüge der Wohltaten werden von Luther theologisch begründet, aber sie sind zugleich auch in der philosophischen Tradition vorhanden. Sowohl Cicero (off 1, 45-60) als auch Seneca (ben 1, 6-15) unterscheiden deutlich zwischen ökonomischen Transaktionen und Pflichten einerseits und freiwilligen Wohltaten andererseits. Die Wohltaten sollen mit Dankbarkeit empfangen werden; die Dankbarkeit ist aber keine Bezahlung, und die Wohltat ist nicht durch Gegenleistung bestimmt, sondern durch die wohlwollende Intention des Gebers. Deswegen sind für Seneca (e.g. ben 4, 30-32) auch einseitige Wohltaten echte Wohltaten. $^{37}$

In seiner Schrift De beneficiis diskutiert Seneca auch ausführlich die Rolle von Gott als Geber und als Vorbild aller menschlichen Wohltaten. Senecas Götter können Wohltaten allen Menschen spenden, auch solchen, die sich unwissend oder ablehnend zu den göttlichen Gaben verhalten:

Was die Götter tun, aller Dinge vorzügliche Urheber, die einem Wohltat ohne dessen Wissen zu erweisen beginnen und auch, ohne Dank zu erhalten, damit fortfahren ... Nichtsdestoweniger jedoch, nach Art wirklich guter Eltern, die zu den Schmähungen ihrer kleinen Kinder lachen, säumen die Götter nicht, Wohltaten denen zuzuwenden, die an dem Urheber der Wohltaten zweifeln ... (ben 7, 31).

Bemerkenswert ist auch, dass Seneca eine lange Liste von natürlichen Wohltaten Gottes anführt, die u.a. die folgenden Sachen umfasst: Bäume, Kräuter, Nahrungsmittel, Lebewesen aller Art, Flüsse, Heilwasserquellen, Landflächen, Metalle, Gold, Silber, Kupfer, Eisen, Himmel am Tag und Nacht, Atem, Licht, Blut, Delikatessen, Reizmittel des Genusses, Ruhe, Frieden, Tierherden, alle Künste und die verschiedenen Lebensalter des Menschen (ben 4, 46). Die Interpretation von Naturereignissen als Wohltaten Gottes unterstützt die These, dass Gott seine Wohltaten allen Menschen ohne Rücksicht auf deren Dankbarkeit erweist. Eine solche Interpretation hat Parallelen schon im Alten Testament (e.g. Psalm 104), aber es ist auffallend, wie Luther und Seneca die kosmischen Ereignisse und den Reichtum der Natur als

\footnotetext{
${ }^{36}$ WA 55/2, 401, 508-525.
}

${ }^{37}$ Siehe z.B. R. SAARINEN, "The Language of Giving in Theology" (NZSTh 52, 2010, 268-301), hier: 278-282, sowie ENGBERG-PEDERSEN, Gift-Giving (s. Anm. 15). 
göttliche Wohltaten auffassen. Auch in seinen späteren Schriften wiederholt Luther oft ähnliche Listen ${ }^{38}$.

Darüber hinaus ist zu notieren, dass Luther in der ersten Psalmenvorlesung eine ähnliche Dreiteilung von Tauschbeziehungen wie Seneca verwendet. (1) Die Verdienste und deren Gerechtigkeit beziehen sich auf den ökonomischen Tausch, also auf Handel. (2) Die Gabenbeziehungen unter den Menschen vollziehen sich im Kreislauf von Wohltaten, bei dem die einzelnen Wohltaten mit angemessener Dankbarkeit empfangen werden. Darüber hinaus sollen (3) die Wohltaten Gottes als kosmischer Ausgangspunkt aller späteren Wohltaten und Dankbarkeit begriffen werden. Die göttlichen Wohltaten beziehen sich universal auf alle Menschen, und zwar dessen ungeachtet, wer sie identifiziert oder dankbar ist. Wenn Luther in der ersten Psalmenvorlesung konstatiert, dass Gottes Sein im Geben besteht, braucht er darum keine platonische Emanationslehre oder kein bestimmtes Partizipationsverständis zu vertreten $^{39}$, sondern der Kontext spricht eher von der herkömmlichen Dreiteilung zwischen Handelsbeziehungen, Gabenbeziehungen und den konstituierenden Wohltaten Gottes. Diese Dreiteilung konnten die Humanisten bei Seneca wiederfinden: Sie ist also ein genereller Topos oder ein Kulturgut, das bei den konkreten Tauschbeziehungen und Wohltaten vorausgesetzt wurde.

In der ersten Psalmenvorlesung verbindet Luther auch eigenständig die Wohltaten mit einem spezifischen theologischen Thema, nämlich mit Gottes Barmherzigkeit. Einen unmittelbaren Anlass für diese Verbindung bietet die Erklärung des Wortes hesed in Reuchlins hebräisches Wörterbuch: „Hesed - Wohltat, Gnade oder Gnadenhaftigkeit. Die? Septuaginta übersetzt das Wort mit eleos, deswegen benutzt die lateinische Übersetzung stets Barmherzigkeit ${ }^{40}$ Die misericordia war ein zentrales Thema der spätmittelalterlichen Frömmigkeit, und das häufige Vorkommen des lateinischen Wortes in den Psalmen motiviert Luther dazu, den Zusammenhang zwischen Wohltaten und Barmherzigkeit theologisch zu reflektieren.

38 Z.B. im Großen Katechismus: BSLK 648. In BSLK 649, 11-17 spricht der lateinische Text von empfangenen Wohltaten. Andere Belege in SAARINEN, Beneficia (s. Anm. 32), 178-181.

${ }^{39}$ Einige positive Äußerungen des jungen Luthers zu Platon brauchen also keine generelle Annahme des Platonismus bedeuten. Vgl. T. DIETER, Der junge Luther und Aristoteles, 2001, 619-627.

40 J. REUCHLIN, De rudimentis hebraicis libri III, Reprint 1974, 185. 
So kann er zum Beispiel konstatieren, die Wohltaten Gottes auf uns seien lauter Barmherzigkeit, die Gott uns ohne Rücksicht auf unsere Verdienste gebe. ${ }^{41}$ Das Wort Barmherzigkeit als Attribut von göttlichen Wohltaten bedeutet, dass Gott allein sie gibt und dass unser Dank nur an Gott gerichtet sein soll. Auch wenn eine undankbare Person eine solche Anerkennung nicht leistet, bekennen die barmherzigen Taten an sich schon den Herrn, so dass andere Menschen durch diese Taten Gott anerkennen können. ${ }^{42}$ Die Verbindung zwischen Wohltaten und Barmherzigkeit prägt auch Luthers spätere Theologie. ${ }^{43}$

Die Tugend der Barmherzigkeit ist biblisch und bringt einen Aspekt in die Diskussion ein, der bei Seneca nicht als solcher vorhanden ist. Auch für Seneca ist aber die Wohltat vor allem durch den guten Willen und die rechte Intention gekennzeichnet. Ein göttlicher Geber betrachtet nach Seneca nicht die Gegenleistung des Empfängers, sondern ausschließlich seine fides, das heißt seine Rezeptivität (solam accipientis fidem specta, ben 3, 14, 2). Auf diese Weise sollen auch die Menschen in den Wohltaten vorgehen:

Die meisten Wohltaten und bedeutendsten wendet uns der Gott wahrhaftig ohne eine Hoffnung zu, seinerseits etwas zu erhalten, da je weder er eine Zuwendung bedarf noch wir ihm irgendetwas zuwenden können; also ist eine Wohltat um ihrer selbst willen eine wünschenswerte Sache. Allein werde an ihr gewünscht des Empfangenden Nutzen; ihm wollen wir nahekommen und zugleich beiseite lassen unseren Vorteil. (ben 4, 9).

In ähnlicher Weise ist die göttliche Barmherzigkeit eine Gabenbeziehung, die nicht die Verdienste oder die Dankbarkeit des Empfängers betrachtet, sondern Gottes grundlegende Wohltat zum Ausdruck bringt. Der theologische Ort der Barmherzigkeit ist nicht im Humanismus, sondern in der heiligen Schrift sowie in der spätmittelalterlichen Frömmigkeit zu finden. Luthers Verbindung von Barmherzigkeit und Wohltaten in der ersten Psalmenvorlesung veranschaulicht gewissermaßen Senecas Einsicht, dass Gottes grundlegende Wohltat alle späteren beneficia vorbereitet. ${ }^{44}$

${ }^{41}$ WA 55/2, 832, 94-96.

${ }^{42}$ WA 55/2, 831, 81-84.

${ }^{43}$ Saarinen, BenEFicia (s. Anm. 32) 181-184.

${ }^{44}$ Gegen HENAFF, Preis (s. Anm. 15), 428-429 sei bemerkt, dass eine solche Theologisierung der Gaben nicht nur eine protestantische Entwicklung, sondern schon ein antiker Gedanke ist. 
3. Doctrina - die Autonomie der Lehre

Die Unterscheidung zwischen Gaben und Handelsobjekten spielt ebenfalls eine große Rolle in der begrifflichen Bestimmung von Lehre und Unterweisung, Themen, die auch für unseren Lutherkongress bedeutsam sind. Natalie Zemon Davis hebt die Wichtigkeit des mittelalterlichen Sprichwortes Scientia est donum dei in verschiedenen Lebensbereichen hervor. ${ }^{45}$ Als Gaben Gottes können das Wissen und die Lehre nicht verkauft werden. Wissen und Lehre sind keine Handelsobjekte, die zum Privatbesitz werden könnten, sondern sie sollen als permanent freie Gaben verbreitet werden, wie die Statuten der Universität Paris im Jahre 1536 formulierten. ${ }^{46}$

Wenn die Professoren in ihrem Unterricht eine Distribution der freien göttlichen Gaben praktizieren, können sie für diese Beschäftigung kein Gehalt und keine Bezahlung erhalten in der Weise, wie ein Handwerker einen Preis für sein Produkt verlangt. Das verbreitete Wissen ist kein Eigentum des Professors in dem Sinne, wie ein Handelsprodukt dem Hersteller oder dem Händler gehört. Das Wissen ist eine frei bleibende Gabe oder eine Leihgabe, die nicht zum Privateigentum des Lehrers wird und deswegen nicht verkauft werden kann. Auf der anderen Seite bleiben die Professoren nicht gänzlich ohne Belohnung für ihr Lehramt, da sie von dankbaren Studenten Honorare und Gaben erhalten können. Zemon Davis bestreitet, dass die Honorare nur maskierte Bezahlungen waren. Für die Akademiker des 16. Jahrhunderts war es wichtig, eine ordentliche Unterscheidung zwischen den Geschäftshandlungen einerseits und der Zirkulation der freien Gaben andererseits zu treffen. ${ }^{47}$ Wenn die Professoren Honorare anstatt von Gehältern erhielten, könnten sie der Natur ihrer Lehre und ihres Wissens als freie göttliche Gabe Rechnung tragen.

Marcel Henaff unternimmt eine philosophiegeschichtliche Vertiefung dieser Einsicht. Nach Henaff entdeckt schon Platons Sokrates den Gedanken, dass die Wahrheit keinen Preis haben kann. „Wissen und Geld lassen sich nicht mit einem Maß messen“, konstatiert Aristoteles in seiner Eudemischen Ethik. ${ }^{48}$ Aus diesem Grund sind die Beziehungen zwischen

${ }^{45}$ ZEMON DAVIS, Gift (s. Anm. 18), 75-81. Das Sprichwort kommt z.B. bei ThOMAS von AQUIN, Summa theol. 1/2 q112 a5 2, vor.

${ }^{46} \mathrm{AaO} ., 79-80$.

${ }^{47}$ AaO., 81; Henaff, Preis (s. Anm. 15), 36-37.

${ }^{48}$ HenAfF, Preis (s. Anm. 15), 159-162. ARISTOTELES, Eudemische Ethik VII, 10; $1243 b 23$. 
Wahrheit, Wissen und Belohnung im abendländischen Denken keine Handelsbeziehungen, sondern Gabenbeziehungen. ${ }^{49}$ Es ist wichtig zu sehen, dass die Gabenbeziehungen auch zumeist reziprok sind: Der Empfänger des Wissens reagiert auf diese Gabe, obwohl er keinen Kaufakt leistet.

Der historische Grundmodus eines solchen Reagierens ist die Dankbarkeit, die auch konkrete Formen wie Honorar oder Gegengabe annehmen kann. Henaff unterscheidet zwischen (1) reziproken zeremoniellen Gaben, die die gegenseitige Anerkennung der Partner zum Zweck haben, und (2) vorwiegend unilateralen Gaben, die die vielen Dimension des griechischen Wortes charis zum Ausdruck bringen: die Großmut, Gunst und Gnade des Gebers, die Dankbarkeit und Freude des Empfängers. Die christliche Tradition des Abendlandes erörtert vor allem diese Aspekte der Gabenbeziehungen. ${ }^{50}$

Zum unmittelbaren Kontext der Dankbarkeit gehört auch der Begriff des Ehrenhaften (honestas, honestum), den Cicero (e.g. off 1, 60-61, 79, 93) und Seneca (e.g. ben 4, 1, 9-10, 16) ausführlich erläutern. Für die kalkulativen Handelsbeziehungen spielt die Ehre nur untergeordnete Rolle, während die Gabenbeziehungen ehrenhaft vorgehen und die Würde des Gebers sowie des Empfängers manifestieren sollen. Zugleich ist Ehre aber auch ambivalent, da der Geber falschen Stolz vermeiden und ohne Rücksicht auf Gegengaben großzügig agieren soll. Die Ehre des Empfängers kann leicht verletzt oder falsch verstanden werden. Ein angemessenes honestum soll sowohl problematische Arroganz als auch falsche Demut vermeiden.

Die wahrhafte Achtung, Dankbarkeit und Freude können nicht gekauft oder verkauft werden, sondern sie kommen durch angemessene Gabenbeziehungen zustande. Zemon Davis präsentiert eine große Zahl an konkreten Beispielen, wie angemessene Gunst, Würde und Ehre durch die sozialen Praktiken von Gabenbeziehungen im 16. Jahrhundert aufgebaut wurden. Die angemessene Ehrung und Würdigung eines akademischen Lehrers ist ein Beispiel für solche Gabenbeziehungen, denn einerseits verteilt der Lehrer besonders wertvolle göttliche Gaben, andererseits kommen diese Gaben nicht aus seinem Privatbesitz und können deswegen nicht verkauft werden. ${ }^{51}$

\footnotetext{
${ }^{49}$ AaO., 36-37, 479-480. Siehe auch ZEMON DAvis, Gift (s. Anm. 18), 73-109.

${ }_{51}^{50} \mathrm{AaO} .$, 406-409.

51 ZEMON DAVIS, Gift (s. Anm. 18), 79-82. Auch ihr Kapitel "Gifts gone Wrong" (aaO., 110-141) beleuchtet das Wesen von Ehre bzw. honestum.
} 
Luthers Texte zur Wahrhaftigkeit der Lehre und der christlichen Predigt nehmen teil an dieser großen Tradition des Wissens als Gabe, die keinen Preis hat. Die Spuren dieser Tradition sind nicht ohne weiteres offenbar, da Luther von der biblischen Sprache ausgehend seine Argumente präsentiert. Wenn ich im Folgenden diese Ähnlichkeiten näher erläutere, meine ich nicht, dass Luthers Argumente ausschließlich die sozialen Praktiken des 16. Jahrhunderts oder die philosophische Tradition des Wissens als Gabe manifestieren. Die theologischen Argumente haben durchaus ihren Eigenwert; es geht vor allem darum, dass diese Argumente gewisse Entsprechungen mit den philosophischen und sozialgeschichtlichen Traditionen der Gabenbeziehungen aufweisen und so der Kommunikation der theologischen Botschaft dienen.

In einer Predigt aus dem Jahre 1537 behandelt Luther das Predigtamt im Anschluss an Matth. 21, 14-16. ${ }^{52}$ Ein Prediger soll nicht ehrgeizig sein, aber die Lehrer und Doktoren leiden häufig an diesem Übel. Weil zeitliche Ehre nur Gott gebührt, ist Ehrgeiz im Predigtamt ein besonders giftiges Laster. ${ }^{53}$ Im Prediger wirken Ehrgeiz und falscher Stolz wie Gift, das sein Amt und seine Lehre unrein macht. Wer im Predigtamt nach Ehre und Weisheit strebt, ist ungläubig. ${ }^{54}$ Ein ehrgeiziger Prediger will sich selbst als feinen Mann darstellen. Wenn die Zuhörer ihn loben, muss er auch sie loben, und das gegenseitige Loben bringt schließlich sowohl den Prediger als auch seine Gemeinde in die Hölle. ${ }^{55}$ Die so beschriebene Reziprozität ist einer Handelsbeziehung ähnlich: Der ehrgeizige Prediger beweist seinen eigenen Wert und verkauft sich so an die eitlen Zuhörer, die als Gegenleistung ebenfalls den Preis des Lobes verlangen.

Gegen Stolz und Eitelkeit soll der Prediger mit den Worten von Joh. 7,18 sagen: „Wer von ihm selbst redet, der sucht seine eigene Ehre. Wer aber sucht die Ehre des, der ihn gesandt hat, der ist wahrhaftig.“ Der rechte Prediger soll ebenfalls Demut üben und sagen: „Geheiligt

${ }^{52}$ WA $47,397-408$.

53 WA 47, 401, 19-26: ,... ein Prediger sol nicht ehrgeitzig sein, wie sich den auch ein jeder Christ also halten sol. Dan ein Christ ist nicht drumb getaufft und zum ewigen leben beruffen, das ehr seine ehre suchete, viel weniger ein Prediger. Dennochs ists ein gemeine plage, das die Doctores und Lehrer der kirchen oben aus und nirgents an wollen, als am Muntzer und andern zu sehen ist. Es ist an einem Christen, jha viel mehr an einem Prediger kein grossere, ergere gifft, dan wen ehr in zeittlichen sachen ehre suchet, welche ehre doch Gott allein geburet.“ - Der traditionelle Gegensatz zwischen „Gabe“ und „Gift" prägt den gesamten hier referierten Abschnitt Luthers.

${ }^{54}$ WA 402, 8-17. 
werde dein Name. ${ }^{\text {656 }}$ Die Dialektik der angemessenen Ehre ist aber komplex, denn auf der anderen Seite soll der Prediger stolz die Ehre des Wortes Gottes verteidigen. Dann sagen aber die Gegner, dieser Prediger suche seine eigene Ehre. Die Frommen sind auch an sich lobenswert, obwohl ein Lob zu falschem Stolz verleiten kann. Angesichts dieser Komplexität vergleicht Luther die falsche Ehre mit einer Distel: Wo immer man sie hinwirft, bleibt sie irgendwo stecken. ${ }^{57}$ Die Wahrhaftigkeit zu predigen, ohne den eigenen Wert und die eigene Ehre ins Bild zu bringen, ist also eine schwierige Tugend. ${ }^{58}$

Die Dialektik der angemessenen Ehre findet aber schließlich eine gelungene Synthese, wenn man das Wort und die damit verbundene Ehre nicht als Privatbesitz betrachtet:

Kompts nun in den Punct, das ich soll gottes wort predigen und bekennen, da mus man getrost stehen und sagen: Alhier ist die warheit und dort die Lugen, und als dan sagen: O himlisscher vater, es ist dein wortt, du wilst, das ichs soll frej bekennen und sagen: 'Geheiliget werde dein name'. So aber andere sagen, ich sej ehrgeitzig und stoltz, so liegen sie dran, den ich suche alsdan die ehre des, der mich gesanth hat, den das wortt ist nicht mein wortt, sondern gottes. Wens mein Wortt were, das ich predigte, so wolt ich meine ehre verfluchen. Aber es ist gottes wort, drumb so hebet man ahn und betet: 'Geheiliget werde dein name' \&c.. und ist stoltz und hoffertig. ${ }^{59}$

In ähnlicher Weise wie ein Professor im 16. Jahrhundert seine Lehre weder selbst erfunden hat noch diese freie Gabe besitzen kann, stellt Luther vom Wort Gottes fest:

Dieses wort hab ich nicht erdacht, ich habs auch nicht geschrieben, nemet brillen in die hand und thut die heilige schrifft auf ... ihr sollet mir dieses Buchs schuler bleiben. Wenn der Papst fragt,willst Du mich lehren, antwortet der Prediger: Ich lehre dich nicht, sondern ich weise dir einen Lehrer ... Gott hat mir das Wort gegeben. ${ }^{60}$ Wenn das Wort als gegeben verstanden wird, bleibt es Gabe und erzeugt nicht den giftigen Stolz, sondern manifestiert die Ehre Gottes.

Zusammenfassend kann Luther das Problem der angemessenen Ehre wie folgt lösen:

${ }^{55}$ WA $402,25-29$.

${ }^{56}$ WA 47, 403, 5-19.

${ }^{57}$ WA 47, 404, $13-405,8$.

58 „Der Wertbegriff ist eigentlich ein Gegenbegriff zum Begriff der Wahrheit“, wie E. JÜNGEL, Wertlose Wahrheit, 1990, 92 konstatiert.

${ }^{59}$ WA 47, 405, 9-17.

${ }^{60}$ WA 47, 405, 22 - 406, 26. 
Man mus alhier von einander scheiden Gottes ehre und menschen ehre. Wens gottes ehre angehet, do sei du nicht demuttig. Hoere andere nicht, die grosser und gelertter sindt den du. Lass es geschehen, das sie dieselbigen gaben haben, aber dennochs sind sie nicht gelerter noch hoher den das Gottliche wortt, welches alleine gottes ist, den dasselbige ist ein meister aller meister. Du bist nicht grosser noch mechtiger den diess

wortt. Du und ich sollen unter dem wort sein. Das Wort ist nicht mein und dein ... ${ }^{61}$

Diese Einsichten Luthers von der Lehre und dem Wort Gottes wenden also den alten Gedanken an, demgemäß das Wissen eine freie Gabe Gottes ist. Folglich kann der Lehrer seinen Unterricht nicht als eigenes Kapital betrachten oder für eigennützige Zwecke anwenden. Als freie Gabe oder Leihgabe kann die Lehre nicht im Rahmen der Handelsbeziehungen, sondern nur im Rahmen der Gabenbeziehungen angemessen verstanden und vermittelt werden.

Gerhard Ebeling hat Luthers Gedanken untersucht, demgemäß die „Lehre“ zu Gott gehört, das „Leben“ dagegen zu uns, wie der Reformator an einer berühmten Stelle des Galaterbriefkommentars konstatiert. ${ }^{62}$ Im Rahmen unserer Fragestellung können die von Ebeling untersuchten Luthertexte so ausgelegt werden, dass das Leben aus messbaren ökonomischen Leistungen und Beiträgen besteht, während die Lehre eine himmlische Gabe manifestiert, die der Mensch nicht mit seinen eigenen Fähigkeiten erfinden oder ändern darf: ... die lere ist Gotts wort und Gotteswarheit selbs, Aber das leben ist unsers thuns mit, Darumb mus die lere gantz rein bleiben, Und wer am leben feilet und gebrechlich ist, da kan Gott wol gedult haben und vergeben, Aber die lere selbs, darnach man leben sol, endern odder auff heben, das kan und wil er nicht leiden, sol es auch nicht leiden, Denn das trifft seine hohe Goettliche maiestet selbs an, Da gilt kein vergeben noch gedult haben. ${ }^{63}$

${ }^{61}$ WA $47,407,18-24$.

${ }^{62}$ WA 40/2, 46, 5-8. G. EBELING, „Lehre und Leben in Luthers Theologie“ (in: ders. Lutherstudien III, 1985, 3-43), hier: 30. Die abendländische Ideengeschichte von doctrina ist erstaunlich wenig untersucht worden, siehe z.B. die Ausführungen im Band Vera Doctrina: zur Begriffsgeschichte der Lehre von Augustinus bis Descartes, hg. P. BÜTTGEN et al., 2009.

${ }^{63}$ WA 30, 343, $30-344,20$. 
Nach Luther gehört unser Leben zu uns, aber die Lehre gehört allein zu Gott. Die Lehre betrifft die allerhöchsten Sachen und kann deswegen nicht unser Eigentum sein; die Reinheit dieser kostbaren Leihgabe soll besonders beachtet werden. ${ }^{64}$

Den biblischen Hintergrund dieses Gedankens bildet Joh. 7,16: „Die Lehre ist nicht mein“. Wenn der Lehrer predigt, ist er letzten Endes nicht das Subjekt oder der Besitzer der Lehre, sondern Gott hat das Wort des Predigers gedacht und ihm gegeben. ${ }^{65}$ Die Logik der Lehre folgt der Logik der Gabe und Leihgabe: Ein Christ soll Gott die Ehre geben für all die Gaben, die er erhalten hat. So soll auch ein gelehrter Mensch zugeben, dass seine Lehre nicht eigentlich die seine ist, sondern die Gottes. ${ }^{66}$ Der Lehrer kann zwar zwischen Menschenwort und Gottes Wort unterscheiden und über menschliche Lehren urteilen, aber er kann nicht über Gottes Wort und die christliche Lehre verfügen, weil diese freien Gaben nicht zum Besitz des Predigers werden können. Als Vermittler der göttlichen Lehre ist der Lehrer darum schließlich ein Schüler Gottes, und sein Amt kann als „Schüleramt“ begriffen werden. ${ }^{67}$

Das mittelalterliche Sprichwort wurde häufig auch in der längeren Form: Scientia est donum Dei, unde vendi non potest wiedergegeben: „Das Wissen ist Gabe Gottes, deswegen kann es nicht verkauft werden“. Dieser Satz wurde in der mittelalterlichen Diskussion um

${ }^{64}$ WA 18, 112, 9-13. Ebeling, LEHRE (s. Anm. 62), 30, verweist im Weiteren auf WA $51,518,21-23 ; 520,21-24$ und WA 37, 669, 1-34.

${ }^{65}$ WA 40/2, 255, 25-31.

${ }^{66}$ WA 23, 743, 25 -744, 14: "Drum ist maxima res contemnere nostram gloriam. Nemo ita utitur donis a deo acceptis in commodum proximi, sed ut utilitas sequatur et habeat voluptatem dran. Ergo 1. geben sie die ehr got irem hern pro magno opere, quod deus dedit filium, quia est mera gratia et inexpressibilis, ergo honor non sit noster. ... Quando ehr datur deo et hoc bene caneretur a nobis, so wuerde die 2. leise auch, wenn die Ehr von uns ist, non est discordia. Ubi humilitas, ibi concordia, Sol. Qui volunt domini esse ex paradiso, non potest pacem habere, quia quisque vult aliquid esse et alios contemnere. Sed si dicit pius: Ego sum doctier te, tu ditior, mea doctrina non est mea nec habeo gloriam inde, sed deo pertinet, si possum tibi cum ea servire, sie sol dir offen stehen semper et omnibus hominibus. Si sic ago cum eo, non fimus discordes, quia do donum meum da hin et dico domini mei esse, nach der ere, nach dem nutz proximi." - Hervorhebungen hier und in der nächsten Anm. von mir.

${ }^{67}$ WA 33, 365, 35 - 366, 40: , ... und ob ich schon kan scheiden die lehre, so von Gott ist oder von menschen hehrkommen, dennoch so hab ich die macht nicht uber das wortt Gottes zu herrsschen oder gottes wortt zuverwerffen, sondern dieweil ich gottes Schuler bin, so werde ich mit meinem Schuler ampt ein Magister uber menschen satzung und lehre, aber nicht uber gottes wortt. ... Also gehets auch mit einem Hausvatter zu, der knecht soll nicht fragen nach des herrn wortt und disputiren, sondern es heist: der Herr hats gesagt, darumb so schweige der knecht stille und thue, was der herr befohln hat. Also ists alhier auch, es will der herr Christus sagen: Jch bin ein prediger und Gott redet selbst, es ist sein wortt, Meine lehre 
kirchliche Ämter und Simonie benutzt ${ }^{68}$; zum Beispiel Pierre d'Ailly erläutert ihn ausführlich in seiner Abhandlung über die Lust. ${ }^{69}$ Auch Luther erwähnt den Endteil des Sprichwortes einmal in seiner Genesisvorlesung, wenn er die Simonie und den Verkauf von geistlichen Gütern kritisiert. $^{70}$

Auf der anderen Seite vertritt Luther bekanntlich die Meinung, dass der Pfarrer eine angemessene Belohnung verdient. Luthers kurze Annotationes zu Matthäus sind aufschlussreich, denn sie zeigen, wie er anhand einer leicht spiritualisierenden Auslegung die Unverfügbarkeit der Lehre mit der Bezahlung des Pfarrers verbinden kann. Obwohl Jesus in Matth. 10, 8 sagt, dass wir umsonst empfangen haben und darum umsonst geben sollen, verbietet dieser Satz nach Luther nicht eine Bezahlung des Predigers nach seinem konkreten Bedarf. Jesus verbietet nur die falsche Gier, aber der Prediger kann sich selbst und seine Familie mit angemessener Bezahlung ernähren. Am wichtigsten ist es zu verstehen, dass ein Lehrer in seinem Amt des Wortes keine innerliche Begierde nach Geld, Ruhm, Ehre oder Macht haben sollte; ein Prediger soll nichts sagen oder tun, um solche eigennützigen Ziele zu fördern. Als Träger des Wortes soll der Lehrer nackt auftreten, das heißt, als frei von bösen Affekten und so, dass seine Hörer nur das Wort und nicht die Person des Predigers wahrnehmen. ${ }^{71}$

Obwohl der Prediger auf diese Weise eine konkrete Bezahlung erhalten kann, bleibt die innere Natur der Lehre als Gabe unberührt. Die Bezahlung ist eine äußerliche Kostenerstattung für den Prediger, der seine eigenen Verdienste in keiner Beziehung zu seiner Lehre setzen soll. Die Hingabe, die Jesus in Matth. 10, 8-10 verlangt, bedeutet für Luther letzten Endes die innere Ablehnung der falschen Affekte, die die Gabe zum Handelsobjekt machen. Der bezahlte Prediger soll aus seiner Person oder Lehre kein Handelsobjekt machen - modern ausgedrückt: er hat kein intellektuelles Recht auf seine Lehre. So bleibt die innerliche Gabenbeziehung zwischen dem Lehrer und seinen Zuhörern auch dann bestehen, wenn der Lehrer eine Entlohnung bekommt.

ist nicht mein, drumb gedenckt und horet, was ich rede.“

${ }^{68}$ Siehe G. POST et al., „,The medieval heritage of a humanistic ideal: 'scientia donum dei est, unde vendi non potest"' (in: Traditio 11, 1955, 195-234).

${ }^{69}$ Eine Edition dieses Textes (d'AILLY, Radix omnium malorum est cupiditas) bietet A. BERnsteIn, Pierre d'Ailly and the Blanchard Affair, 1978; hier: 199.

${ }^{70}$ WA 43, 281, 22-33.

${ }^{71}$ WA 38, 496, 5-28. 
Eine wichtige systematisch-theologische Anwendung des Gedankens vom Wissen als Gabe besteht darin, dass die Offenbarung Gottes in einem solchen Kontext als unverfügbare Selbstoffenbarung verstanden wird. Christoph Schwöbel formuliert diesen Sachverhalt treffend:

Die für die reformatorische Theologie grundlegende Unterscheidung und Beziehung zwischen Gottes Werk und menschlichem Handeln greift auch in Bezug auf das Verhältnis von Offenbarung und kirchlichem Amt ... Die Autorität der Offenbarung kann nicht auf die Kirche übertragen werden. Sie ist Zeugin der Wahrheit, indem sie Auslegerin des Wortes ist und auf die nur von Gott selbst verliehene Gabe der inneren Klarheit hofft. $^{72}$

Dabei ist allerdings wichtig zu sehen, dass auch das römisch-katholische Lehramt auf seine Weise dem mittelalterlichen Prinzip „scientia est donum dei“ sowie dem biblischen Satz „die Lehre ist nicht mein“ Rechnung trägt. Wenn der katholische Amtsträger konstatiert, dass er keine Befugnis hat, die für ihn als Leihgabe übertragene Lehre zu ändern, will er auch den Gabencharakter der Offenbarung bewahren. So ist der konfessionelle Unterschied nicht einfach in der Eigentumsbeziehung zwischen Amt und Offenbarung, sondern eher in den unterschiedlichen Interpretationen des gemeinsamen Satzes „Die Lehre ist nicht mein“ feststellbar.

\section{Accipere - die Passivität des Empfangens}

Die Freiheit des Menschen im Empfangen des Evangeliums gehört zu den großen Streitfragen des abendländischen Christentums. Im Rahmen unseres Themas können wir die folgende Frage stellen: Kann eine Unterscheidung zwischen Handelsbeziehungen und Gabenbeziehungen auch die Freiheit bzw. Unfreiheit des Christen neu gestalten und somit für die Lösung dieser Streitfrage hilfreich sein? Ich möchte im Folgenden eine vorsichtige bejahende Antwort geben. Zugleich ist eine Warnung nötig, denn die Frage ist sehr kompliziert, und unterschiedliche Spuren führen leicht zu unterschiedlichen Ergebnissen.

${ }^{72}$ C. SchwÖBEL, Art. Offenbarung V2b, RGG4, Bd. 6, 479-481, hier: 480. 
In der Lutherforschung der letzten Jahre hat vor allem Bo Kristian Holm ${ }^{73}$ sehr wertvolle Anregungen zur Beantwortung dieser Frage gegeben. Holm will Luthers Theologie konsistent im Rahmen der Gabenbeziehungen analysieren. Er gebraucht sozialwissenschaftliche Studien, die die Gegenseitigkeit von Gaben hervorheben. Holm will zeigen, dass eine gewisse Reziprozität oder menschliche Mitwirkung auch in der Heilsaneignung bei Luther zu bemerken ist, obwohl diese Gegenseitigkeit deutlich von allerlei Selbstgerechtigkeit und Handelsbeziehungen unterschieden werden soll. Die Einsicht, dass Gaben ihrer eigenen Logik der Gegenseitigkeit folgen, motiviert Holm dazu, menschliche Mitwirkung im Rahmen der Gabenbeziehung theologisch zu explizieren. ${ }^{74}$

Die Sprache von Gaben, Dankbarkeit und Wohltaten, also von Gabenbeziehungen insgesamt, führt allerdings in Sachen Mitwirkung und Synergie m.E. eher zu einer antipelagianischen und monergistischen Heilslehre. Zwar ist es auch wichtig zu sehen, dass Geben und Empfangen stets einen intentionalen und personalen Empfänger voraussetzen; deswegen ist eine gewisse Reziprozität mit der Begrifflichkeit der Gabe semantisch verbunden. Aber das große Bild, nämlich der frühneuzeitliche Unterschied zwischen „Gifts and Sales“, Gabenbeziehungen und Handelsbeziehungen, führt zu einer angemessenen Minimalisierung der menschlichen Mitwirkung im Empfangen des Heils.

In Luthers unmittelbarer Umgebung herrschte die spätmittelalterliche Handlungstheorie, für die die Möglichkeit der willentlichen Zustimmung konstitutiv für die Entstehung der menschlichen Handlung ist. Ohne solche Zustimmung kann der Mensch seine Handlung nicht als die „seine“ bezeichnen, sondern sie wird zu einem bloßen Geschehen oder kompulsiven Verhalten. Luthers Lehrer Usingen gebraucht die Handlungstheorie Johannes Buridans, um die herkömmliche Lehre von der menschlichen Handlung zu entfalten. Nach Usingen und Buridan ist die willentliche Zustimmung zu einer Handlung ein actus acceptationis oder refutationis, und der moralische Wert der Handlung wird normalerweise durch die Art dieser Zustimmung gemessen. ${ }^{75}$

Die buridanische Handlungstheorie weist auffallende Ähnlichkeiten mit einer Handelsbeziehung auf. Um dominus actus sui, Herr seiner Akte bzw. ein Moralagent zu sein,

${ }^{73}$ B. K. HoLM, Gabe und Geben bei Luther (TBT 134), 2006

${ }^{74}$ Holm, aaO.,104-131. Vgl. auch B. K. HoLM, „,Rechtfertigung und Gabe: ein Beitrag zur Resozialisierung der Rechtfertigungslehre" (ÖR 60, 2011, 178-190).

${ }^{75}$ Siehe ausführlicher SAARINEN, Weakness (s. Anm. 5), 36-41, 106-108. 
muss das Subjekt durch seine willentliche Zustimmung ein dominium, ein Machtverhältnis, zum entstehenden Akt aufweisen. Die in dieser Zustimmung entstehende acceptatio oder refutatio ist wie die Annahme oder Nicht-Annahme eines käuflichen Angebotes, das dem Subjekt bereitgestellt wird. Bei Buridan und Usingen geschieht diese Bereitstellung als ein vorausgehender, unfreier Akt der complacentia oder displicentia, die die Sympathie bzw. Antipathie des potentiellen „Käufers“ erwecken soll. Das Subjekt hat aber eine freie Wahl darin, seine faktische Zustimmung zu bestimmten Akten unter vielen bereitgestellten Handlungsoptionen zu geben. ${ }^{76}$

Wichtig für unser Thema ist, dass die so dargestellte Handlungstheorie wie ein Kaufakt auf dem Marktplatz aussieht: Das Subjekt sieht eine Reihe von bereitgestellten Optionen, die ihm mehr oder weniger gefallen und so, wie die Spätscholastik formuliert, sub ratione boni erscheinen. Danach gibt das Subjekt seine willentliche Zustimmung zu bestimmten Optionen, die der freie Wille aus dem Gesamtangebot wählt. Die Zustimmung ist somit wie ein Kaufvertrag, und die moralische Natur der Person wird anhand dieses Kaufvertrags gemessen. Durch diese Theorie, die Usingen als „katholische“ Handlungstheorie bezeichnet $^{77}$, ist der Zustimmungsakt und folglich das gesamte „Menschenwerk“ einer Handelsbeziehung auffallend ähnlich.

Wenn Martin Luther mit seiner theologischen Sprache von Gabe, Wohltat und Passivität eine bewusste Alternative zu dieser seines Erachtens pelagianisch klingenden Handlungstheorie entwerfen will, findet er wichtige Unterstützung aus einigen Aspekten der klassischen humanistischen Sprache des Gebens und Empfangens. Die entscheidenden lateinischen Wörter bei Cicero und Seneca sowie in de Vulgata sind bekanntermaßen dare und accipere. Dabei hat aber accipere im Kontext der Gabenbeziehungen einen grundlegend anderen Sinn als die Begriffe consentire (zustimmen), acceptatio und refutatio in der spätmittelalterlichen Handlungstheorie.

Für das abendländische Christentum sind vor allem Matth. 10,8 und 1 Kor. 4,7 ausschlaggebende Belegstellen für das rechte Empfangen. Gratis accepistis, gratis date, „Umsonst habt ihr's empfangen, umsonst gebt es auch“ formuliert Matth. 10,8 prägnant; 1. Kor. 4,7 fragt rhetorisch: Quid autem habes quod non accepisti, „Was hast du, was du nicht

${ }^{76}$ SAARINEN, aaO., 36-41, 106-108. In meinen früheren Veröffentlichungen spreche ich jedoch nicht von einer „Handelsbeziehung“.

${ }^{77}$ SAARINEN, aaO., 106-107. 
empfangen hast?" Beide Verse betonen, dass die christliche Existenz sich im Rahmen der Gabenbeziehungen, als Geben und Empfangen vollzieht. Bekanntlich war 1. Kor. 4,7 für Augustin ein ganz wichtiger Vers bei der Bekämpfung des Pelagianismus. ${ }^{78}$ Empfangen, accipere, signalisiert also ein Verhalten, das ohne eigene Leistung geschieht.

Sowohl Cicero als auch Seneca diskutieren das rechte Empfangen von Wohltaten. Während beide die Freiwilligkeit der Wohltaten hervorheben, betont Cicero die aktive Reziprozität und die gegenseitigen Pflichten (e.g. off 1, 47-48). Für Seneca ist aber auch ein passives Empfangen von Wohltaten durchaus möglich, weil eigentlich nur die zielgerichtete Intention des Gebers für die Wohltat konstitutiv ist. Die angemessene Reaktion des Empfängers ist zwar wichtig für die Wahrnehmung seiner Würde, aber das Wesen der Wohltat ist nicht an sich von dieser Reaktion abhängig. Im rechten Geben einer Wohltat soll ich Seneca zufolge beachten, „dass ich um dessentwillen handle, in dessen Händen ich die Wohltat gelangen lassen will, ihn für würdig halte, ihm das gerne schenke und aus meinem Geschenk Freude gewinne“،. (ben 4, 29, 3). Im Idealfall reagiert der Empfänger mit Dankbarkeit und zeigt so die vorausgesetzte Würde.

Allerdings ist diese Würde ein komplexes Phänomen, das nicht notwendigerweise ein Danksagen oder sonstiges Reagieren des Empfängers bedeutet. Die Intentionalität des Gebers braucht nicht von einer ähnlichen Intentionalität und Willentlichkeit des Empfängers begegnet werden; bisweilen kann der Geber die Würde auch ohne Dankbarkeit oder sonstige Reaktion feststellen. „Eine Wohltat kann jemand empfangen, ohne es zu wissen“, konstatiert Seneca (ben 6, 8, 1). Es ist sogar möglich, gegen den ausgesprochenen Willen des Empfängers eine Wohltat zu erweisen: Eltern, die ihre Kinder bisweilen mit Zwang erziehen, sind ein Beispiel dafür. „Daher sind unter den Wohltaten am größten die, die wir von den Eltern empfangen haben, ohne es zu wissen oder zu wollen“ (ben 6, 24, 2). Das Modell solcher Wohltaten ist für Seneca ein göttliches Wesen, das die Würde und den Bedarf der Empfänger besser als sie selbst feststellen kann. Unter den Wohltaten des göttlichen Wesens ist gerade die am bedeutendsten, die einem Menschen, der sich dagegen beklagt, erwiesen wird (ben 6, 23, 8). Die Götter säumen nicht, Wohltaten denen zuzuwenden, die an dem Urheber der Wohltaten zweifeln (ben 7, 31, 4).

\footnotetext{
${ }^{78}$ So E. MüHLENBERG, Art. Augustin, RGG4, Bd 1, 959-967, hier: 965.
} 
Senecas Diskussion ist für uns relevant, weil er ein Modell des Empfangens entwirft, das sehr deutlich vom scholastischen Zustimmungsmodell der menschlichen Handlung abweicht. Das Zustimmungsmodell setzt voraus, dass der Mensch das präsentierte Angebot selber wählt. Das Wohltatenmodell Senecas lehrt aber, dass im Geben und Empfangen nur der Geber eigentlich willentlich und intentional tätig sein muss. Das Empfangen kann ohne oder sogar gegen den Willen des Empfängers stattfinden - auch in solchen Fällen kann man von accipere sprechen. Zugleich muss der Empfänger aber eine solche Würde haben, dass er als Person ein Adressat des Gebensaktes sein kann. Bisweilen konzipiert Seneca diese Einsicht anhand des Gedankens der Verzögerung: Der Empfänger soll zu einem späteren Zeitpunkt die Mündigkeit haben, dankbar zu sein (ben 7, 30-32). Wichtig ist aber, dass die in den Wohltaten ausgesprochene Gabenbeziehung eine ganz andere Art des Empfangens aufweist als die Handelsbeziehung, für die die willentliche Zustimmung beider Seiten konstitutiv ist. Für eine Wohltat ist es ausreichend, wenn nur der Geber einen zielgerichteten Willensakt zustande bringt.

Für Luthers Rede von Gottes Wohltaten ist bekanntermaßen die Passivität des menschlichen Empfangens, die sogenannte mere passive, von grundlegender Bedeutung. In einer Diskussion mit Bo Holm hat Ingolf Dalferth neulich Wörter wie „Passivitätsaktivität“ und „Empfangenkönnen der Gabe“ benutzt, wenn er mere passive interpretiert hat. ${ }^{79}$ Philipp Stoellger hat gezeigt, dass die Kategorie der passio für Luther eine facettenreiche Wirklichkeit darstellt, die die gleichzeitige Möglichkeit von Ruhe und Offenheit durchaus erlaubt. ${ }^{80}$ Mein Plädoyer für ein besonderes accipere, willenloses Empfangen, das in den Gabenbeziehungen zum Vorschein kommt, geht in die gleiche Richtung wie die wertvollen Studien von Dalferth und Stoellger. ${ }^{81}$ Meine These ist, dass die humanistische Philosophie der Wohltaten, besonders wie sie in De beneficiis formuliert worden ist, eine bemerkenswerte Ähnlichkeit mit Luthers Einsicht von mere passive aufweist.

Wenn wir Luthers Gebrauch des Wortes accipere näher studieren, fällt sofort auf, dass dieses Empfangen nicht mit dem freien Willen oder mit Werken identifiziert wird, sondern im Gegenteil als glaubendes Empfangen der Wohltaten Gottes. Wer Glauben in Christus

${ }^{79}$ I. U. DALFERTH, „Mere Passive. Die Passivität der Gabe bei Luther“ (in: WordGift-Being, ed. B. K. Holm \& P. WidmanN, 2009, 43-73), hier: 57, 64. Vgl. Holm, Gabe (s. Anm. 73.

${ }^{80}$ P. STOELlger, Passivität aus Passion (HUTh 56), 2010, z.B. 298-308. 
empfängt, ist gerecht und heilig. ${ }^{82}$ Wenn wir im Glauben beten, verspricht Gott, dass wir alles empfangen. ${ }^{83}$ Der Glaube empfängt die Sündenvergebung. ${ }^{84}$

Die humanistische Unterscheidung zwischen Handelsbeziehungen und Gabenbeziehungen kommt an den zahlreichen Stellen zu Vorschein, in denen Luther eine bewusste Unterscheidung zwischen facere und accipere vornimmt: „Lex gibt's facere, fides accipere $^{، 85}$. Unter den weltlichen Menschen wird derjenige gelobt, der viel tut, aber bei Gott derjenige, der vieles empfängt. ${ }^{86}$ Im Feiern des Sakraments wird das Gute nicht von den Menschen gemacht, sondern von ihnen empfangen. ${ }^{87}$ Die Gnade wird nicht so gepredigt: „Mach dies, mach jenes“, sondern so: „Empfange dies, dann hast du es““. ${ }^{8}$

Typisch für diesen Kontrast ist im Weiteren, dass Luther facere als Handlung Gottes und accipere als Reaktion des Christen bezeichnet. „Was Christus lehrt und tut, wird von uns empfangen““ ${ }^{89}$ Das Evangelium fordert nicht unser Tun, sondern unser Empfangen. Gott ist der Handelnde, nicht wir. ${ }^{90}$ In der Nächstenliebe geht es um das Empfangen der für diese Person vollbrachten Liebestaten. ${ }^{91}$ Das Wort Gottes, das Evangelium, wird nicht von uns gemacht, sondern empfangen. ${ }^{92}$ „Wir sollen nichts anderes tun als alles umsonst empfangen“. ${ }^{93}$ Im Neuen Testament werden wir nicht im Tun unterrichtet, sondern im Empfangen, so dass wir alles als Gabe erhalten. ${ }^{94}$ Unser Hören des Evangeliums ist kein Werk, sondern ein Empfangen. „Wenn ich empfange, tue ich nichts. Wenn ich arbeite, empfange ich nicht““.95 „Gott handelt und wir empfangen““.96

${ }^{81}$ Vgl. SAARINEN, Language (s. Anm. 37), 290-294.

${ }^{82}$ WA 39/1, 221, 15.

${ }^{83}$ WA $14,579,6$.

${ }^{84}$ WA $30 / 3,489,10$.

${ }^{85}$ WA 40/1, 426, 2.

${ }^{86}$ WA $1,78,21$.

${ }^{87}$ WA $6,521,30-31$.

${ }^{88}$ WA $11,215,31-32$.

${ }^{89}$ WA 9, 515, 25.

${ }^{90}$ WA $16,586,4-6$.

${ }^{91}$ WA 20, 514, 1-5.

${ }^{92}$ WA $27,136,3-5$.

${ }^{93}$ WA $31 / 2,318,11$.

${ }^{94}$ WA 34/2, 164, 24-26.

${ }^{95}$ WA 36, 15, 10-11. Der Kontext (WA 36, 14, 11-16, 11) erläutert die Dialektik von Handelsbeziehung und Gabenziehung: , Dilige proximum, benefac illi \&c.. Das heyst das gesecz abgeczirckelt ab Euangelio. Das uns heyst thun, Euangelion nihil mandat et exigit, Es heyst nur nhemen und lassen geben, scilicet promissione dei. Das schencke ich dyr, do kan 
Die Belegstellen von accipere zeigen deutlich, dass das Empfangen kein Werk ist, sondern eine willenlose und zugleich personale Rezeption der Wohltaten und Gaben. Der Begriff des Empfangens gehört mit der göttlichen Gabenbeziehung eng zusammen und wird häufig mit solchen Handelsbeziehungen kontrastiert, die facere benutzen. Auf diese Weise ist eine auffallende Ähnlichkeit zwischen Seneca und Luther im Gebrauch des Begriffs accipere zu bemerken. Übrigens wird der scholastische Begriff acceptatio von Luther ganz deutlich als Werk, als facere, verstanden; wie bei Buridan und Usingen setzt dieser Begriff bei Luther eine willentliche Zustimmung voraus. Deswegen kann Luther, anders als die Buridanisten, acceptatio eigentlich nur als göttlichen Akt bezeichnen. ${ }^{97}$ Das Wort consentire, ,zustimmen“, gehört bei Luther ebenfalls eng mit facere zusammen. ${ }^{98}$

Das Verständnis des menschlichen Empfangens als willenlose, personale Gabenbeziehung kann auch hilfreich für die Interpretation von De servo arbitrio sein. Zwar soll Luthers Position in dieser Schrift anhand anderer Begrifflichkeiten skizziert werden ${ }^{99}$, aber sie manifestieren die gleiche Logik der Gabenbeziehungen wie der Kontrast zwischen facere und accipere. In seiner Debatte mit Erasmus nimmt Luther bekanntlich eine ganz eigenwillige Definition von Notwendigkeit als Unveränderlichkeit (immutabilitas) vor. Der Wille kann, so Luther, nicht autonom seine Grundrichtung verändern und ist in diesem Sinne gebunden bzw. unterliegt einer Notwendigkeit. ${ }^{100}$ Allerdings gibt Luther zu, dass (1) der Mensch eine aptitudo naturalis, eine personale rezeptive Fähigkeit hat. ${ }^{101}$ Die Aufgaben dieser Fähigkeit

ich nichts zw thun, Ut baptizari aliquem non est opus meum, sed dei donum et opus, ders thut und schenckt. Summa: per legem exigitur, Durchs Euangelion werde ich zur spende geruffen. Illud discrimen ita notate. Euangelion est donum, do sol ich uffhalden. Den magna est differentia inter Nhemen und geben. Quando accipio, nihil facio. Quando laboro, tunc non accipio. Das eyne ist promissio, aliud praeceptum. Illud dat, illud exigit. Wie eyn lehenher gibt eynem edelman eyn gut, hoc est opus principis. Deinde cum ille ministraverit myt pferden, do thut er was. Das ist de caussa finali. Wens yns geystliche werk kumpt, das gesecz, tunc exigit, terret, si non fecerimus, habemus malam conscienciam. Econtra euangelion praedicat donum, do wyl das hercz nicht hinan."

${ }^{96}$ WA 36, 229, 11.

${ }^{97}$ Z.B. WA 39/1, 228, 9; WA 40/1, 233, 25.

${ }^{98}$ Z.B. WA 1, 472, 26; 2, 592, 5; 8, 116, 33 und 120, 34-35; 12, 177, 10; 40/3, 716, 20; 43, 74, 31 .

${ }^{99}$ Das Wort accipere bezieht sich in De servo arbitrio zumeist auf die philologische Frage, wie ein Wort „verstanden“ (accipitur) wird.

${ }^{100}$ WA $18,634,14-29$.

${ }^{101}$ WA $18,636,16-22$. 
sind denjenigen Funktionen auffallend ähnlich, die bei Seneca sowie in vielen anderen Lutherschriften mit dem Verb accipere bezeichnet worden sind. ${ }^{102}$

Diese zwei Züge erlauben durchaus, dass der Mensch Eindrücke empfangen und diesen mit seinem Willen folgen kann. Luthers Definition von Notwendigkeit verbietet nur die autonome oder spontane Veränderung der Willensrichtung; eine heteronome Bestimmung kann aber bestehen und willentlich unterstützt werden. Im Weiteren gibt Luther zu, dass (2) der Gebrauch einiger unwichtiger Mittel im alltäglichen Leben frei bleibt und dass (3) der Christenmensch anhand der Gnade zu einer gewissen Kooperation mit Gott fähig ist. ${ }^{103}$

Aus diesen Grundzügen entsteht ein handlungstheoretisches Modell, in dem der Motivationsgrund bzw. das Herz des Menschen immer schon durch die empfangenen Eindrücke entweder vom Fleisch oder vom Geist heteronom bestimmt wird. Zugleich ist auch das endgültige Ziel seiner Handlungen durch diese heteronomen Kräfte vorbestimmt. Zwischen dem inneren Motivationsgrund und dem endgültigen Ziel kann der Mensch zwar gewisse unwichtige Mittel frei wählen, aber der Wille kann seine grundlegende Gesamtrichtung nicht autonom verändern. Zugleich ist aber auch wahr, dass der Mensch durchaus willentlich handelt, weil er seinen grundlegenden Eindrücken folgt und gewissermaßen eine Kooperation mit denen aufweist. ${ }^{104}$

Ein derartiges Gesamtbild kann zum beträchtlichen Teil als Gabenbeziehung verstanden werden. Für das Entstehen einer Gabenbeziehung braucht man keinen Willen, wohl aber die aptitudo naturalis bzw. die rezeptive Fähigkeit des personalen Empfangens. Die Gabe hat im Weiteren die Fähigkeit, den Empfänger zu verändern; Senecas Beispiel dafür ist die Erziehung als Gabe, die gegen den Widerstand der Kinder wirksam werden kann. Ingolf Dalferth spricht in diesem Kontext sogar von einer „Gabe zur Destruktion“, um zu zeigen, wie die Gabe der Annihilation die fleischliche Existenz vernichten kann. ${ }^{105}$ Wichtig ist aber, dass bei einer solchen Zerstörung das Person-Sein des Menschen nicht gänzlich aufhört. Die Rolle von aptitudo naturalis sowie der alltäglichen Wahl der unwichtigen Mittel kann so

${ }^{102}$ Auch die rezeptive Fähigkeit ist letzten Endes von Gott gegeben, vgl. WA 18, 753, 8-10 im Anschluss an Joh 3,27 Vulgata: ,non potest homo accipere quicquam nisi fuerit ei datum de caelo". Wichtig ist, dass diese Fähigkeit den Tieren, Pflanzen und leblosen Dingen nicht gegeben worden ist und dass der Mensch auf diese Weise eine Person ist. Die Gaben sowie die Akte von dare und accipere beziehen sich auf Personen.

${ }^{103}$ WA $18,638,4-11 ; 753-754$.

${ }^{104}$ Siehe ausführlicher SAARINEN, Weakness (s. Anm. 5), 128-130. 
interpretiert werden, dass sie die kontinuierliche Identität der Person auf dem heteronom bestimmten Wege vom Fleisch zum Geist durch Annihilation und Umwandlung bewahren.

So verstanden geht es Luther in De servo arbitrio nicht um metaphysische Notwendigkeiten, sondern um die bleibende Passivität des Menschen innerhalb der Gabenbeziehung. Für die Handelsbeziehung ist es konstitutiv, dass der Wille eine mutabilitas, eine autonome Veränderungspotenz, besitzt, damit er durch seine Zustimmung den Kaufvertrag schließen kann. In der Gabenbeziehung reicht es aber, dass das Empfangen als aptitudo naturalis gesichert ist und dass die Identität der Person durch diese Offenheit und die Wahl von unwichtigen Mitteln erhalten bleibt. In der Gabenbeziehung handelt der Mensch ohne autonome Entscheidungspotenz, aber durchaus willentlich, da sein Wille durch die fleischlichen oder geistlichen Gaben heteronom aktiviert wird. Zugleich erlaubt die rezeptive Fähigkeit, dass der empfangende Glaube ${ }^{106}$ und die Affekte des Menschen „seine“ personalen Eigenschaften bleiben, obwohl sie aus theologischer Sicht eher ein accipere als facere oder liberum arbitrium manifestieren.

Diese Vorschläge, Luthers De servo arbitrio im Rahmen der humanistischen Gabenbeziehung zu interpretieren, sind provisorischer Art, da Luthers Text eine philosophische Systematik eher vermeidet. In vielen anderen Schriften bietet jedoch Luthers explizite und systematische Kontrastierung zwischen accipere und facere eine starke Evidenz für die These, dass die humanistische Dialektik zwischen ,gifts and sales“, Gabenbeziehungen und Handelsbeziehungen, in Luthers Theologie ein systematisches Deutungsmuster ist, das seine Auffassung von den menschlichen Werken tiefgreifend prägt.

\section{Fazit}

Anhand der neuen Studien von Natalie Zemon Davis und Marcel Henaff habe ich die These formuliert, dass die Unterscheidung zwischen Gabenbeziehungen und Handelsbeziehungen zu Luthers Zeit eine prominente geistesgeschichtliche Idee war. Diese Unterscheidung war

${ }^{105}$ DAlferth, Mere Passive (s. Anm. 79), 54.

${ }^{106}$ Wegen seiner rezeptiven Fähigkeit ist der Glaube für Luther ein Ergreifen der theologischen Wirklichkeit. Zu dieser sog. fides apprehensiva siehe R. SAARINEN, Art. Glaube (in: Luther-Lexikon, hg. V. LEPPIN, erscheint 2013). 
vor allem durch die popularphilosophischen Werke Ciceros und Senecas den Gelehrten bekannt, aber sie prägte auch das kulturelle Verhalten der verschiedenen Klassen in der Gesellschaft. Ich habe nachgewiesen, dass Luther diese Unterscheidung in verschiedenen Variationen benutzt, um seine theologische Botschaft zu erhellen. Die Wohltaten Gottes, die Autonomie der christlichen Lehre und die Passivität des menschlichen Empfangens sind theologische Phänomene, die die grundlegende Figur der Gabenbeziehung manifestieren. Zugleich kritisiert Luther das Verstehen des Gottesverhältnisses als eine Handelsbeziehung.

Wenn Luther die Unterscheidung von „gifts and sales“ benutzt, geht es ihm allerdings nicht primär um die Vertretung einer philosophischen oder sozialgeschichtlichen Position, sondern die Unterscheidung dient der theologischen Kommunikation: Luther will zeigen, wie seine reformatorische Theologie anhand von Gabenbeziehungen verstanden werden kann. Die von Luther gebrauchte Terminologie bietet starke Evidenz dafür, dass sein Gebrauch der Unterscheidung zur Wirkungsgeschichte von Ciceros De officiis und Senecas De beneficiis gehört. Allerdings ist eine direkte literarische Abhängigkeit schwierig nachzuweisen. Da wir aus anderen Studien wissen, dass diese Texte zu Luthers Zeit weit verbreitet waren und die Unterscheidung an sich zur sozialen und intellektuellen Kultur Europas gehörte, können wir Luther auch ohne direkte literarische Abhängigkeit dieser Wirkungsgeschichte zurechnen.

Dieser Befund vertieft die Einsicht von Helmar Junghans, der gemäß Luther viele positive Einflüsse aus dem Humanismus seiner Zeit erhalten hat. Er zeigt im Weiteren, dass Luthers philosophischer Hintergrund nicht nur im Aristotelismus der Spätscholastik zu lokalisieren ist. Auch die humanistische Rezeption von Cicero und Seneca spielt für den Reformator eine Rolle, die kultur- und sozialgeschichtlich beträchtlich sein kann. Durch die Begrifflichkeit von Lehre und Handel kann diese Rezeptionslinie auch die frühneuzeitlichen Auffassungen von Pädagogik und Ökonomie beleuchten. 